\title{
Blow-up or global existence for the fractional Ginzburg-Landau equation in multi-dimensional case
}

\author{
Luigi Forcella, Kazumasa Fujiwara, Vladimir Georgiev, and Tohru Ozawa
}

\begin{abstract}
The aim of this work is to give a complete picture concerning the asymptotic behaviour of the solutions to fractional Ginzburg-Landau equation. In previous works, we have shown global well-posedness for the past interval in the case where spatial dimension is less than or equal to 3. Moreover, we have also shown blow-up of solutions for the future interval in one dimensional case. In this work, we summarise the asymptotic behaviour in the case where spatial dimension is less than or equal to 3 by proving blow-up of solutions for a future time interval in multidimensional case. The result is obtained via ODE argument by exploiting a new weighted commutator estimate.
\end{abstract}

\section{Introduction}

In this paper, we consider the following complex Ginzburg - Landau (CGL) equation in a future time interval

Luigi Forcella

Bâtiment des Mathématiques, École Polytechnique Fédérale de Lausanne, Station 8, CH-1015

Lausanne, Switzerland e-mail: luigi.forcella@epfl.ch

Kazumasa Fujiwara

Centro di Ricerca Matematica Ennio De Giorgi, Scuola Normale Superiore, Piazza dei Cavalieri, 3, 56126 Pisa, Italy e-mail: kazumasa.fujiwara@sns.it

Vladimir Georgiev

Department of Mathematics, University of Pisa, Largo Bruno Pontecorvo 5 I - 56127 Pisa, Italy, Faculty of Science and Engineering, Waseda University, 3-4-1, Okubo, Shinjuku-ku, Tokyo 1698555, Japan, and IMI-BAS, Acad. Georgi Bonchev Str., Block 8, 1113 Sofia, Bulgaria e-mail: georgiev@dm.unipi.it

Tohru Ozawa

Department of Applied Physics, Waseda University, 3-4-1, Okubo, Shinjuku-ku, Tokyo 169-8555, Japan e-mail: txozawa@waseda.jp 


$$
\begin{cases}i \partial_{t} u+D u=i|u|^{p-1} u, & t \in[0, T), \quad T>0, \quad x \in \mathbb{R}^{n}, \\ u(0, x)=u_{0}(x), & x \in \mathbb{R}^{n},\end{cases}
$$

where $u$ is a complex valued unknown function, $p>1$, and $D=(-\Delta)^{1 / 2}$. The choice of $D$ is closely connected with the recent attempts to develop fractional quantum mechanical approach (see [23] ).

We shall observe some new interesting phenomena. On one hand, if we take a future time interval as in (1), then we shall obtain a blow-up result. If, instead, we take past time interval $(-T, 0], T>0$ in the place of the future time interval, then global small data existence for (1) can be proved and therefore we have a similarity to a diffusion type process.

Before giving the main results on the local and global well-posedness for (1), we introduce some notations. For a Banach space $X$ and $1 \leq p \leq \infty$ let $L^{p}\left(\mathbb{R}^{n} ; X\right)$ be a $X$-valued Lebesgue space of $p$-th power. We abbreviate $L^{p}\left(\mathbb{R}^{n} ; \mathbb{C}\right)$ as $L^{p}\left(\mathbb{R}^{n}\right)$. For $f, g \in L^{2}\left(\mathbb{R}^{n}\right)$, we define the inner product as

$$
\langle f, g\rangle_{L^{2}\left(\mathbb{R}^{n}\right)}=\int_{\mathbb{R}^{n}} f(x) \bar{g}(x) d x .
$$

For $s \in \mathbb{R}$, let $H^{s}\left(\mathbb{R}^{n}\right)$ be the usual inhomogeneous Sobolev space defined as $H^{s}\left(\mathbb{R}^{n}\right)=(1-\Delta)^{-s / 2} L^{2}\left(\mathbb{R}^{n}\right)$. Let $\dot{H}^{s}\left(\mathbb{R}^{n}\right)$ be the usual homogeneous Sobolev space defined as $\dot{H}^{s}\left(\mathbb{R}^{n}\right)=(-\Delta)^{-s / 2} L^{2}\left(\mathbb{R}^{n}\right)$. $H_{\text {rad }}^{s}\left(\mathbb{R}^{n}\right)$ is the restriction to radial functions of $H^{s}\left(\mathbb{R}^{n}\right)$. Lip refers to space of Lipschitz functions on euclidean space. For $f, g: A \subseteq \mathbb{R}^{n} \rightarrow[0, \infty), f \lesssim g$ means that there exists $C>0$ such that for any $a \in A$ $f(a) \leq C g(a)$. Given two Banach spaces $X, Y, Y \hookrightarrow X$ means that $Y \subset X$ with continuous embedding. Moreover, we say that a Cauchy problem is locally well-posed forward in time in $X$, if for any $X$-valued initial data, there exists $T>0$ and a Banach space $Y \hookrightarrow C([0, T] ; X)$ such that there exists a unique solution to the Cauchy problem in $Y$ and $\left\|u_{n}-u\right\|_{Y} \rightarrow 0$ as $\left\|u_{0, n}-u_{0}\right\|_{X} \rightarrow 0$, where $u_{n}$ and $u$ are solutions for the Cauchy problem for initial data $u_{0}$ and $u_{0, n}$, respectively (the last property goes under the name of continuous dependence on the initial data). We also say that a Cauchy problem is globally well-posed forward in time in $X$ if the Cauchy problem is locally well-posed for any $T>0$. Moreover, we also say that a Cauchy problem is globally well-posed in $X$ with sufficiently small data, if we have the property above for sufficiently small data with respect to the $X$-norm.

Let us notice that equation (1) is invariant under the scale transformation

$$
u_{\lambda}(t, x)=\lambda^{1 /(p-1)} u(\lambda t, \lambda x)
$$

with $\lambda>0$. Then

$$
\left\|u_{0, \lambda}\right\|_{\dot{H}^{s}\left(\mathbb{R}^{n}\right)}=\lambda^{1 /(p-1)+s-n / 2}\left\|u_{0}\right\|_{\dot{H}^{s}\left(\mathbb{R}^{n}\right)}
$$

and with

$$
s=s_{n, p}:=n / 2-1 /(p-1)<n / 2,
$$


$\dot{H}^{s}$ norm of initial data is also invariant, for this $s_{n, p}$ is called scale critical exponent. We also call $p_{n, s}=1+2 /(n-2 s)$ the $H^{s}\left(\mathbb{R}^{n}\right)$ scaling critical power. For any $s$, in the scaling subcritical case where $p<p_{n, s}$ or $s>s_{n, p}$, (1) is expected to have local solution for any $H^{s}\left(\mathbb{R}^{n}\right)$ initial data on the analogy of scaling invariant Schrödinger equation. For instance, we refer the reader to $[4,6,5,16,17]$. However, with power type nonlinearity without gauge invariance, semirelativistic equations could be not locally well-posed even in scaling subcritical case, see [10].

Here we recall local well-posedness results. It is worth mentioning that Borgna and Rial [2] showed that in one dimensional case, CGL equation with cubic nonlinearity is locally well-posed in $H^{s}(\mathbb{R})$ with $s>1 / 2$. They constructed local solutions by a contraction argument based on the unitarity of the propagator and the Sobolev embedding $H^{s}(\mathbb{R}) \hookrightarrow L^{\infty}(\mathbb{R})$. Similarly, local solutions may be constructed in the case where uniform control of solutions holds, namely, in $H^{s}\left(\mathbb{R}^{n}\right)$ with $s>n / 2$. On the other hand, for fixed $p, s_{n, p}<n / 2$; therefore, the local well-posedness of (1) is expected in wider Sobolev spaces. Indeed, we have the following results that can be established using the approach in [12]:

Proposition 1 ([12]). Let $n=2$. For $p>1$ and $3 / 4<s<p<p_{2, s}$, the Cauchy problem (1) is locally well-posed in $H^{s}\left(\mathbb{R}^{2}\right)$.

Proposition 2 ([12]). Let $n \geq 3$ and $u_{0}$ be radial. For $1<p<p_{n, 1}=1+\frac{2}{n-2}$, the Cauchy problem (1) is locally well-posed in $H_{\mathrm{rad}}^{1}\left(\mathbb{R}^{n}\right)$.

Proposition 3 ([12]). Let $n=3$ and $u_{0}$ be radial. For $p=p_{3,1}=3$, the Cauchy problem (1) is locally well-posed in $H_{\mathrm{rad}}^{1}\left(\mathbb{R}^{3}\right)$ with sufficiently small $H_{\mathrm{rad}}^{1}\left(\mathbb{R}^{3}\right)$ data.

Remark 1. In Proposition 3, since the local existence result is based on a priori estimate of type

$$
\|u\|_{X_{\mathrm{rad}}^{1}(0, T)} \leq C_{0}+C_{1}\|u\|_{X_{\mathrm{rad}}^{1}(0, T)}^{4}
$$

with $C_{1}$ which is independent of $T$, we restrict well-posedness to the small initial data.

We recall that in three dimensional case, $p=p_{3,1}=3$ is a critical value in view of the result in [18]. However, the result in [18] treats non-gauge invariant nonlinearities having constant sign, for which the test function method works. The question of the existence of local and global solutions for $n \geq 3$ and $p \geq 1+2 /(n-2)$ seems, at the best of our knowledge, still open.

Proposition 1 may be justified by a Strichartz estimate introduced by Nakamura and Ozawa in [26] or Ginibre and Velo [14]. We remark that they introduced the estimate to study Klein-Gordon equation and it was sufficient to consider Klein-Gordon equation in scaling subcritical case (see Lemma 1 below). On the other hand, for (1), local solutions cannot be constructed based on their Strichartz estimates in general subcritical case. Therefore, in order to consider the well-posedness in $H^{1}\left(\mathbb{R}^{n}\right)$ for $n \geq 3$, we put radial assumption and apply another Strichartz estimate introduced in [1] by the third author, Bellazzini and Visciglia. For details, see Section 2. 
Next, we review the known blow-up result. In [11], the authors studied the blowup of solutions to (1) in one dimensional case, by an ordinary differential equation (ODE) argument. In order to review their argument, we define a function space $h L^{2}\left(\mathbb{R}^{n}\right)$ by

$$
h L^{2}\left(\mathbb{R}^{n}\right)=\left\{f: \text { mesurable and }\left\|\frac{1}{h} f\right\|_{L^{2}\left(\mathbb{R}^{n}\right)}<\infty\right\},
$$

where $h$ is a mesurable function. In their argument, an ordinary differential inequality (ODI) for the $h L^{2}(\mathbb{R})$ norm of solutions with some $h$ are shown. In particular, we have the following:

Proposition 4. Let $h$ be a Lipschitz function satisfying $1 / h \in L^{\infty}(\mathbb{R}) \cap L^{2}(\mathbb{R})$ and

$$
\left\|\frac{1}{h(\cdot)} \int_{\mathbb{R}}\langle\cdot-y\rangle^{-2} h(y) f(y) d y\right\|_{L^{2}(\mathbb{R})} \leq C\|f\|_{L^{2}(\mathbb{R})} .
$$

Let $u_{0} \in L^{2}(\mathbb{R})$ satisfy

$$
\left\|\frac{1}{h} u_{0}\right\|_{L^{2}(\mathbb{R})} \geq C_{1}^{\frac{1}{p-1}}\left\|\frac{1}{h}\right\|_{L^{2}(\mathbb{R})}
$$

where $C_{1}=\|1 / h \cdot[D, h]\|_{L^{2}(\mathbb{R}) \rightarrow L^{2}(\mathbb{R})}$. If there is a solution $u \in C\left([0, T) ; h L^{2}(\mathbb{R})\right)$ to (1), then

$$
\left\|\frac{1}{h} u(t)\right\|_{L^{2}(\mathbb{R})} \geq e^{-C_{1} t / 2}\left(\left\|\frac{1}{h} u_{0}\right\|_{L^{2}(\mathbb{R})}^{-p+1}+C_{1}^{-1}\left\|\frac{1}{h}\right\|_{L^{2}(\mathbb{R})}^{-p+1}\left\{e^{-C_{1}(p-1) t / 2}-1\right\}\right)^{-\frac{1}{p-1}} .
$$

Therefore, the lifespan is estimated by

$$
T \leq-\frac{2}{p-1} C_{1}^{-1} \log \left(1-C_{1}\left\|\frac{1}{h}\right\|_{L^{2}(\mathbb{R})}^{p-1}\left\|\frac{1}{h} u_{0}\right\|_{L^{2}(\mathbb{R})}^{-p+1}\right) .
$$

Moreover, by scaling argument, the following statement is shown.

Corollary 1 ([11, Corollary 1]). If $p<3$, then any solutions to (1) with non trivial $L^{2}(\mathbb{R})$ initial data cannot stay in $L^{2}(\mathbb{R})$ globally.

Remark 2. In the Corollary above, $p<3$ stands for the condition in one dimensional case of the Fujita exponent generally defined in $\mathbb{R}^{n}$ by $p_{F}:=1+2 / n$ (see also Corollary 2). Then the assumption of Corollary 1 is rewritten by $p<p_{F}$. Under this assumption, by scaling $h$, (3) holds for any non trivial $L^{2}(\mathbb{R})$ initial data $u_{0}$.

Remark 3. Condition (2) was required to guarantee the commutator estimate:

$$
\|[D, h] f\|_{L^{2}(\mathbb{R})} \leq C\|f\|_{L^{2}(\mathbb{R})}, \quad \forall f \in L^{2}(\mathbb{R}) .
$$

We remark that Lenzmann and Schikorra [24, Theorem 6.1] showed that (5) holds for any Lipschitz function $h$, therefore, the assumption (2) can be omitted. 
The commutator estimate (5) implies blow-up for solutions to (1) in the following manner. Let $v(t, x)=u(t, x) / h(x)$, where $u$ is a solution to (1). Then, a straight computation shows that $v$ satisfies

$$
\begin{aligned}
i \partial_{t} v+D v+\frac{1}{h}[D, h] v & =i \frac{1}{h} \partial_{t} u+\frac{1}{h} D u \\
& =i \frac{1}{h}|u|^{p-1} u \\
& =i|h|^{p-1}|v|^{p-1} v
\end{aligned}
$$

Therefore,

$$
\begin{aligned}
\frac{d}{d t}\|v(t)\|_{L^{2}(\mathbb{R})}^{2} & =2 \operatorname{Re}\left\langle v(t), \partial_{t} v(t)\right\rangle_{L^{2}(\mathbb{R})} \\
& =-2 \operatorname{Im}\left\langle v(t), i \partial_{t} v(t)\right\rangle_{L^{2}(\mathbb{R})} \\
& =-2 \operatorname{Im}\left\langle v(t),-D v(t)-\frac{1}{h}[D, h] v(t)+i|h|^{p-1}|v(t)|^{p-1} v(t)\right\rangle_{L^{2}(\mathbb{R})} \\
& =2\left\|\left.|| h\right|^{(p-1) /(p+1)} v(t)\right\|_{L^{p+1}(\mathbb{R})}^{p+1}+2 \operatorname{Im}\left\langle v(t), \frac{1}{h}[D, h] v(t)\right\rangle_{L^{2}(\mathbb{R})} .
\end{aligned}
$$

By the Hölder inequality,

$$
\|v(t)\|_{L^{2}(\mathbb{R})} \leq\left\|\frac{1}{h}\right\|_{L^{2}(\mathbb{R})}^{(p-1) /(p+1)}\left\||h|^{(p-1) /(p+1)} v(t)\right\|_{L^{p+1}(\mathbb{R})},
$$

which together with (7) implies

$$
\frac{d}{d t}\|v(t)\|_{L^{2}(\mathbb{R})}^{2} \geq\left\|\frac{1}{h}\right\|_{L^{2}(\mathbb{R})}^{-p+1}\|v(t)\|_{L^{2}(\mathbb{R})}^{p+1}-\left\|\frac{1}{h}[D, h]\right\|_{L^{2}(\mathbb{R}) \rightarrow L^{2}(\mathbb{R})}\|v(t)\|_{L^{2}(\mathbb{R})}^{2} .
$$

Estimate (8) and Lemma 7 in Section 3 imply that if (3) holds and

$$
\left\|\frac{1}{h} \cdot[D, h]\right\|_{L^{2}(\mathbb{R}) \rightarrow L^{2}(\mathbb{R})}<\infty,
$$

then $\|v(t)\|_{L^{2}(\mathbb{R})}=\|u(t) / h\|_{L^{2}(\mathbb{R})}$ blows-up at a finite time. Therefore, if there exists $1 / h \in L^{2}(\mathbb{R})$ satisfying (9), then the argument above works and blow-up of solutions to (1) is shown. In [11], (9) was shown by the boundedness assumption of $1 / h$ and (5). We remark that (5) holds in more general situation; for example, in multidimensional case. We also remark that in [9] a generalization of (5) taking the form

$$
\left\|\left[(-\mathscr{A})^{1 / 2}, h\right]\right\|_{L^{2}\left(\mathbb{R}^{n}\right) \rightarrow L^{2}\left(\mathbb{R}^{n}\right)} \leq C\|h\|_{\dot{B}_{\infty, 1}^{1}}
$$

is shown, where $\dot{B}_{\infty, 1}^{1}$ is the standard homogeneous Besov space and

$$
\mathscr{A}:=-\nabla \cdot A \nabla+V \text {. }
$$


Here $A$ is a smooth positive-definite $n \times n$ matrix and the real-valued potential $V$ satisfies some weak integrability conditions. On the other hand, $h \in \mathrm{Lip}$ is a natural condition for (5). However, there exists some Lipschitz function $h$ satisfying $1 / h \in$ $L^{2}\left(\mathbb{R}^{n}\right)$ only when $n=1$. This means, we cannot consider blow-up phenomena in multi dimensional case based on (5).

In this paper, we show (9) with polynomial weights which are not Lipschitz in general. In particular, we show the following estimate:

Proposition 5. Let $n \geq 1$ and $n / 2<q<n / 2+1$. Then $\langle\cdot\rangle^{-q}\left[D,\langle\cdot\rangle^{q}\right]$ is bounded operator on $L^{2}\left(\mathbb{R}^{n}\right)$, where $\langle\cdot\rangle=\left(1+|x|^{2}\right)^{1 / 2}$.

Remark 4. Obviously, if $n \geq 1$ and $n / 2<q<n / 2+1$, then $\langle\cdot\rangle^{-q} \in L^{2}\left(\mathbb{R}^{n}\right)$. Moreover, only when $n=1, q$ can be 1 .

Then, we have the following blow-up statement:

Proposition 6. Let $n \geq 1$ and $n / 2<q<n / 2+1$. Let $u_{0} \in\langle\cdot\rangle^{q} L^{2}\left(\mathbb{R}^{n}\right)$ satisfy

$$
\left\|\langle x\rangle^{-q} u_{0}\right\|_{L^{2}\left(\mathbb{R}^{n}\right)} \geq C_{2}^{\frac{1}{p-1}}\left\|\langle x\rangle^{-q}\right\|_{L^{2}\left(\mathbb{R}^{n}\right)}
$$

where

$$
C_{2}=\left\|\langle\cdot\rangle^{-q}\left[D,\langle\cdot\rangle^{q}\right]\right\|_{L^{2}\left(\mathbb{R}^{n}\right) \rightarrow L^{2}\left(\mathbb{R}^{n}\right)} .
$$

If there is a solution $u \in C\left([0, T) ;\left\langle\cdot{ }^{q} L^{2}\left(\mathbb{R}^{n}\right)\right)\right.$ to $(1)$, then

$$
\begin{aligned}
& \left\|\langle\cdot\rangle^{-q} u(t)\right\|_{L^{2}\left(\mathbb{R}^{n}\right)} \\
& \geq e^{-C_{2} t / 2}\left(\left\|\langle\cdot\rangle^{-q} u_{0}\right\|_{L^{2}\left(\mathbb{R}^{n}\right)}^{-p+1}+C_{2}^{-1}\left\|\langle\cdot\rangle^{-q}\right\|_{L^{2}\left(\mathbb{R}^{n}\right)}^{-p+1}\left\{e^{-(p-1) C_{2} t / 2}-1\right\}\right)^{-\frac{1}{p-1}} .
\end{aligned}
$$

Therefore, the lifespan is estimated by

$$
T \leq-\frac{2}{p-1} C_{2}^{-1} \log \left(1-C_{2}\left\|\langle\cdot\rangle^{-q}\right\|_{L^{2}\left(\mathbb{R}^{n}\right)}^{p-1}\left\|\langle\cdot\rangle^{-q} u_{0}\right\|_{L^{2}\left(\mathbb{R}^{n}\right)}^{-p+1}\right) .
$$

Corollary 2. Let $n \geq 1$. If $p<p_{F}:=1+2 / n$, then any solutions to (1) with non trivial $L^{2}\left(\mathbb{R}^{n}\right)$ initial data cannot exist globally.

Remark 5. As Remark 2, under the condition, $p<p_{F}$, by scaling $h$, (10) holds for any non trivial $L^{2}\left(\mathbb{R}^{n}\right)$ data.

In [11], so as to show (5), higher frequency part of $D$ is handled by the CoifmanMeyer estimate and lower frequency part is estimated by (2). We remark that (5) is regarded as a Kato-Ponce inequality. For related subjects, we refer the reader to $[15,19,20,25]$ and we remark that Fourier multiplier argument plays a critical role in these references. On the other hand, it seems not easy to obtain (9) based on a Fourier multiplier argument because of the weight function. Therefore, we show Proposition 5 by using the following representation of the commutator: 


$$
\left(\left[D,\langle\cdot\rangle^{q}\right] f\right)(x)=C \cdot \mathrm{P} . \mathrm{V} \cdot \int_{\mathbb{R}^{n}} \frac{\left(\langle x\rangle^{q}-\langle x+y\rangle^{q}\right) f(x+y)}{|y|^{n+1}} d y,
$$

where P.V. stands for Principal Value (for detail, we refer the reader to [8]). Combining (13) and the Calderón-Zygmund theory, we show (9) with non-Lipschitz weight functions.

Our next step is to study the global existence result for negative times of the following Cauchy problem:

$$
\begin{cases}i \partial_{t} u+D u=i|u|^{p-1} u, & t \in(-T, 0], \quad T>0, \quad x \in \mathbb{R}^{n}, \\ u(0, x)=u_{0}(x), & x \in \mathbb{R}^{n} .\end{cases}
$$

Making the change of variables $t \rightarrow-t$, we reduce this problem to the future time interval for the Cauchy problem

$$
\begin{cases}i \partial_{t} u-D u=-i|u|^{p-1} u, & t \in[0, T), \quad T>0, \quad x \in \mathbb{R}^{n}, \\ u(0, x)=u_{0}(x), & x \in \mathbb{R}^{n} .\end{cases}
$$

At least formally, (15) may be rewritten in the following integral equation:

$$
u(t)=U(-t) u_{0}-\int_{0}^{t} U\left(-t+t^{\prime}\right)\left|u\left(t^{\prime}\right)\right|^{p-1} u\left(t^{\prime}\right) d t^{\prime},
$$

where $U(t)=e^{i t D}$.

Then, Propositions 1, 2, and 3 are valid for (15). Moreover, for (16), we can obtain the following a priori estimates that we include for completeness but detailed proofs can be found in [12].

Proposition 7 ([12]). Let $n \in \mathbb{N}$ and $p>1$. Let $u_{0} \in L^{2}\left(\mathbb{R}^{n}\right)$ and $T>0$. Let $u \in$ $L^{\infty}\left(0, T ; L^{2}\left(\mathbb{R}^{n}\right)\right) \cap L^{p}\left(0, T ; L^{2 p}\left(\mathbb{R}^{n}\right)\right)$ be a solution to the integral equation (16) for the initial data $u_{0}$. Then, for any $t_{1}, t_{2}$ with $0<t_{1}<t_{2}<T$,

$$
\left\|u\left(t_{2}\right)\right\|_{L^{2}\left(\mathbb{R}^{n}\right)}^{2}+2\|u\|_{L^{p+1}\left(t_{1}, t_{2} ; L^{p+1}\left(\mathbb{R}^{n}\right)\right)}^{p+1}=\left\|u\left(t_{1}\right)\right\|_{L^{2}\left(\mathbb{R}^{n}\right)}^{2} .
$$

Proposition 8 ([12]). Let $n \in \mathbb{N}$ and $p>1$. Let $u_{0} \in H^{1}\left(\mathbb{R}^{n}\right)$ and $T>0$. Let $u \in$ $L^{\infty}\left(0, T ; H^{1}\left(\mathbb{R}^{n}\right)\right) \cap L^{p-1}\left(0, T ; L^{\infty}\left(\mathbb{R}^{n}\right)\right)$ be a solution to the integral equation (16) for the initial data $u_{0}$. Then, for any $t_{1}, t_{2}$ with $0 \leq t_{1}<t_{2} \leq T$,

$$
\begin{aligned}
& \left\|\nabla u\left(t_{2}\right)\right\|_{L^{2}\left(\mathbb{R}^{n}\right)}^{2}+2\left\||u|^{\frac{p-1}{2}} \nabla u\right\|_{L^{2}\left(t_{1}, t_{2} ; L^{2}\left(\mathbb{R}^{n}\right)\right)}^{2}+\frac{p-1}{2}\left\||u|^{\frac{p-3}{2}} \nabla|u|^{2}\right\|_{L^{2}\left(t_{1}, t_{2} ; L^{2}\left(\mathbb{R}^{n}\right)\right)}^{2} \\
& =\left\|\nabla u\left(t_{1}\right)\right\|_{L^{2}\left(\mathbb{R}^{n}\right)}^{2} .
\end{aligned}
$$

Proposition 9 ([12]). Let $n=1,2, p>1, n / 2<s<\min \{2, p\}$, and $T>0$. Let $u_{0} \in H^{s}\left(\mathbb{R}^{n}\right)$ and $u \in L^{\infty}\left(0, T ; H^{s}\left(\mathbb{R}^{n}\right)\right) \cap L^{2}\left(0, T ; L^{\infty}\left(\mathbb{R}^{n}\right)\right)$ be a solution to (16) for the initial data $u_{0}$. Then for any $t_{1}, t_{2}$ with $0<t_{1}<t_{2}<T$, 


$$
\left\|u\left(t_{2}\right)\right\|_{\dot{H}^{s}\left(\mathbb{R}^{n}\right)}^{2} \leq\left\|u\left(t_{1}\right)\right\|_{\dot{H}^{s}\left(\mathbb{R}^{n}\right)}^{2}+C \int_{t_{1}}^{t_{2}}\|u(t)\|_{L^{\infty}\left(\mathbb{R}^{n}\right)}^{p-1}\|u(t)\|_{\dot{H}^{s}\left(\mathbb{R}^{n}\right)}^{2} d t .
$$

Proposition 10 ([12]). Let $1 \leq n \leq 3, u_{0} \in H^{2}\left(\mathbb{R}^{n}\right)$ and $T>0$. Let $u \in C\left((0, T) ; H^{2}\left(\mathbb{R}^{n}\right) \cap\right.$ $L^{\infty}\left(\mathbb{R}^{n}\right)$ ) be a solution to the integral equation (16) for the initial data $u_{0}$. Then, for any $t_{1}, t_{2}$ with $0<t_{1}<t_{2}<T$,

$$
\begin{aligned}
& \left\|u\left(t_{2}\right)\right\|_{\dot{H}^{2}\left(\mathbb{R}^{n}\right)}^{2}+2 \sum_{j, k=1}^{n} \int_{t_{1}}^{t_{2}}\left\|u(t) \partial_{j} \partial_{k} u(t)\right\|_{L^{2}\left(\mathbb{R}^{n}\right)}^{2} d t \\
& \leq\left\|u\left(t_{1}\right)\right\|_{\dot{H}^{2}\left(\mathbb{R}^{n}\right)}^{2}+2 n^{2}(n+1) \int_{t_{1}}^{t_{2}}\|u(t)\|_{\dot{H}^{1}\left(\mathbb{R}^{n}\right)}^{4-n}\|u(t)\|_{\dot{H}^{2}\left(\mathbb{R}^{n}\right)}^{n} d t .
\end{aligned}
$$

Therefore, for (14) we have the following:

Proposition 11. Under the conditions of Propositions 1, 2, and 3, (14) is globally well-posed.

This paper is composed as follows. In Section 2, we show local well-posedness of (1) by means of Strichartz estimates of [1, 14, 26]. In Section 3, blow-up for (1) is shown with a weighted commutator estimate. In Section 4, a priori estimates for (14) are shown by a direct approach leading to the global well-posedness results.

\section{Local well-posedness of (1)}

This section is devoted to the proof of the local well-posedness for the Cauchy problem of (1), where $u_{0}(x)=u(0, x)$ is considered as initial datum. The proof is essentially the same as [12] but for the reader's convenience, we give a proof for Propositions 1, 2, and 3. Here we consider the corresponding integral equation:

$$
u(t)=\Phi(u)(t)=U(t) u_{0}+\int_{0}^{t} U\left(t-t^{\prime}\right)\left|u\left(t^{\prime}\right)\right|^{p-1} u\left(t^{\prime}\right) d t^{\prime} .
$$

where $U(t)=e^{i t D}$.

\subsection{Two dimensional case}

In two dimensional case, the local well-posedness may be obtained by the following Strichartz estimates:

Lemma 1 ([26, Lemma 2.1], [14, Remark 3.2]). Let $\left(q_{1}, r_{1}\right)$ and $\left(q_{2}, r_{2}\right)$ satisfy

$$
\frac{1}{r_{j}}=\frac{1}{2}-\frac{2}{q_{j}}, \quad 2 \leq r_{j} \leq \infty, \quad 4 \leq q_{j} \leq \infty
$$


for $j=1,2$. Then for $s \in \mathbb{R}$,

$$
\begin{aligned}
& \|U(t) \phi\|_{\left.L^{q_{1}\left(0, T ; B_{r_{1}}\right.}{ }^{s-\frac{3}{q_{1}}}\left(\mathbb{R}^{2}\right)\right)} \lesssim\|\phi\|_{H^{s}\left(\mathbb{R}^{2}\right)},
\end{aligned}
$$

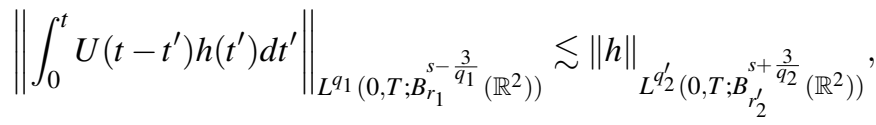

where $B_{p}^{s}\left(\mathbb{R}^{2}\right)=B_{p, 2}^{s}\left(\mathbb{R}^{2}\right)$ is the usual inhomogeneous Besov space.

Lemma 2 ([12, Lemma 3.2]). Let $r>2$, and $T>0$. If

$$
s>\frac{3}{4}+\frac{1}{2 r},
$$

then $B_{r}^{s-\frac{3}{2}\left(\frac{1}{2}-\frac{1}{r}\right)}\left(\mathbb{R}^{2}\right) \hookrightarrow L^{\infty}\left(\mathbb{R}^{2}\right)$.

We can now proceed with the proof of Proposition 1.

Proof (Proof of Proposition 1). At first we fix $3 / 4<s<p<p_{2, s}$. Let $\left(q_{1}, r_{1}\right)$ satisfy the conditions of Lemma 1, Lemma 2 and $q_{1}>p-1$. We remark that such a pair exists under the assumption $s<p<p_{2, s}$. Let $X^{s}(0, T)=L^{\infty}\left(0, T ; H^{s}\left(\mathbb{R}^{2}\right)\right) \cap$ $L^{q_{1}}\left(0, T ; B_{r_{1}}^{s-3 / q_{1}}\left(\mathbb{R}^{2}\right)\right)$. Then, for a fixed $T$,

$$
\begin{aligned}
\|\Phi(u)\|_{X^{s}(0, T)} & \leq\left\|u_{0}\right\|_{H^{s}\left(\mathbb{R}^{2}\right)}+C\left\||u|^{p-1} u\right\|_{L^{1}\left(0, T ; H^{s}\left(\mathbb{R}^{2}\right)\right)} \\
& \leq\left\|u_{0}\right\|_{H^{s}\left(\mathbb{R}^{2}\right)}+C T^{1-(p-1) / q_{1}}\|u\|_{X^{s}(0, T)}^{p}
\end{aligned}
$$

and

$$
\begin{aligned}
& \|\Phi(u)-\Phi(v)\|_{X^{s}(0, T)} \\
& \leq C\left\|\left|\left\|\left.u\right|^{p-1} u-|v|^{p-1} v\right\|_{L^{1}\left(0, T ; H^{s}\left(\mathbb{R}^{2}\right)\right)}\right.\right. \\
& \leq C T^{1-(p-1) / q_{1}}\left(\|u\|_{X^{s}(0, T)}+\|v\|_{X^{s}(0, T)}\right)^{p-1}\|u-v\|_{X^{s}(0, T)} \\
& +C T^{1-(p-1) / q_{1}}\left(\|u\|_{X^{s}(0, T)}+\|v\|_{X^{s}(0, T)}\right)^{\max (1, p-1)}\|u-v\|_{X^{s}(0, T)}^{\min \{1, p-1\}} .
\end{aligned}
$$

This means that if $T$ is sufficiently small, then $\Phi$ is a map from

$$
B_{X^{s}(0, T)}\left(2\left\|u_{0}\right\|_{H^{s}\left(\mathbb{R}^{2}\right)}\right):=\left\{f \in X^{s}(0, T) \mid\|f\|_{X^{s}(0, T)} \leq 2\left\|u_{0}\right\|_{H^{s}\left(\mathbb{R}^{2}\right)}\right\} .
$$

into itself. Moreover, if $p \geq 2, \Phi$ is a contraction map in $X^{s}(0, T)$. If $p<2, \Phi$ may not be a contraction map on $X^{s}(0, T)$ for any $T>0$. On the other hand, it is not difficult to see that

$$
\begin{aligned}
& \|\Phi(u)-\Phi(v)\|_{L^{\infty}\left(0, T ; L^{2}\left(\mathbb{R}^{2}\right)\right)} \\
& \lesssim T^{1-(p-1) / q_{1}}\left(\|u\|_{X^{s}(0, T)}+\|v\|_{X^{s}(0, T)}\right)^{p-1}\|u-v\|_{L^{\infty}\left(0, T ; L^{2}\left(\mathbb{R}^{2}\right)\right)} .
\end{aligned}
$$


Therefore (20) and (21) imply that if $u_{1} \in B_{X^{s}(0, T)}\left(2\left\|u_{0}\right\|_{H^{s}\left(\mathbb{R}^{2}\right)}\right)$ and $u_{k}=\Phi\left(u_{k-1}\right)$ for $k \geq 2$, then there exists $u^{*}=\lim _{k \rightarrow \infty} u_{k}$ in $L^{\infty}\left(0, T ; L^{2}\left(\mathbb{R}^{2}\right)\right)$. Since $\Phi\left(u_{k}\right) \rightarrow$ $\Phi\left(u^{*}\right)$ in $L^{\infty}\left(0, T ; L^{2}\left(\mathbb{R}^{2}\right)\right)$ as $k \rightarrow \infty, u^{*}$ is a solution of (19). Moreover, since $X^{s}(0, T) \hookrightarrow L^{\infty}\left(0, T ; H^{s}\left(\mathbb{R}^{2}\right)\right), u^{*}$ is also in $L^{\infty}\left(0, T ; H^{s}\left(\mathbb{R}^{2}\right)\right)$, which and (20) imply

$$
u^{*} \in L^{q_{1}}\left(0, T ; B_{r_{1}}^{s-\frac{3}{q_{1}}}\left(\mathbb{R}^{2}\right)\right) .
$$

If $s>1$, by the Gagliardo-Nirenberg inequality, for some $0<\theta<1$,

$$
\|u-v\|_{L^{\infty}\left(0, T ; L^{\infty}\left(\mathbb{R}^{2}\right)\right)} \lesssim\|u-v\|_{L^{\infty}\left(0, T ; L^{2}\left(\mathbb{R}^{2}\right)\right)}^{\theta}\|u-v\|_{L^{\infty}\left(0, T ; H^{s}\left(\mathbb{R}^{2}\right)\right)}^{1-\theta}
$$

and therefore the solution map depends continuously on the initial data in $H^{s}\left(\mathbb{R}^{2}\right)$. In the case where $s \leq 1$, by (21), the solution map depends continuously on the initial data in $L^{2}\left(\mathbb{R}^{2}\right)$. We define $s_{3}, s_{4}>0$ so that they satisfy the following:

$$
\begin{aligned}
& \max \left\{\frac{3}{4}+\frac{1}{2 r_{1}}, s_{4}-\frac{3}{4}(p-1)\right\}<s_{3}<s_{4}<\min \left\{s, s_{3}+\frac{3}{4}\right\}, \\
& r_{3}=\frac{3}{2}\left(s_{3}-s_{4}+\frac{3}{4}\right)^{-1},
\end{aligned}
$$

and $q_{3}=\frac{3}{s_{4}-s_{3}}$, where $\left(q_{3}, r_{3}\right)$ satisfy the condition of Lemma 1 . Let $u$ and $v$ be solutions of (1) for initial data $u_{0}$ and $v_{0}$, respectively. Then by Lemma 1 ,

$$
\begin{aligned}
& \left.\|u-v\|_{L^{q_{1}}\left(0, T ; B_{r_{1}}\right.}^{s_{3}-\frac{3}{q_{1}}}\left(\mathbb{R}^{2}\right)\right) \\
& \leq\left\|u_{0}-v_{0}\right\|_{H^{s_{3}}\left(\mathbb{R}^{2}\right)}+C\left\|\left.|| u\right|^{p-1} u-|v|^{p-1} v\right\|_{L^{q_{3}^{\prime}}\left(0, T ; B_{r_{3}^{\prime}}^{s_{4}}\left(\mathbb{R}^{2}\right)\right)} .
\end{aligned}
$$

For $z_{j} \in \mathbb{C}$ with $j=1,2,3,4$, with $w_{1}=z_{2}-z_{1}$ and $w_{2}=z_{4}-z_{3}$,

$$
\begin{aligned}
& \left|z_{4}\right|^{p-1} z_{4}-\left|z_{3}\right|^{p-1} z_{3}-\left|z_{2}\right|^{p-1} z_{2}+\left|z_{1}\right|^{p-1} z_{1} \\
& =\frac{p+1}{2} \int_{0}^{1}\left|z_{3}+\theta w_{2}\right|^{p-1} d \theta w_{2}-\frac{p+1}{2} \int_{0}^{1}\left|z_{1}+\theta w_{1}\right|^{p-1} d \theta w_{1} \\
& +\frac{p-1}{2} \int_{0}^{1}\left|z_{3}+\theta w_{2}\right|^{p-3}\left(z_{3}+\theta w_{2}\right)^{2} d \theta \overline{w_{2}} \\
& -\frac{p-1}{2} \int_{0}^{1}\left|z_{1}+\theta w_{1}\right|^{p-3}\left(z_{1}+\theta w_{1}\right)^{2} d \theta \overline{w_{1}} .
\end{aligned}
$$

Then a direct computation implies that 
Blow-up or global existence for fGL in multi-dimensional case

$$
\begin{aligned}
& \left.|| z_{4}\right|^{p-1} z_{4}-\left|z_{3}\right|^{p-1} z_{3}-\left|z_{2}\right|^{p-1} z_{2}+\left|z_{1}\right|^{p-1} z_{1} \mid \\
& \lesssim\left(\left|z_{3}\right|^{p-1}+\left|z_{4}\right|^{p-1}\right)\left|w_{2}-w_{1}\right| \\
& +\frac{p+1}{p}\left|w_{1}\right|\left|z_{3}-z_{1}\right|^{p-1}+\frac{1}{p}\left|w_{1}\right|\left|z_{4}-z_{2}\right|^{p-1}+\left(\left|z_{3}\right|^{p-1}+\left|z_{4}\right|^{p-1}\right)\left|w_{2}-w_{1}\right| \\
& +\left|w_{1}\right|\left|z_{3}-z_{1}\right|^{p-1}+\left|w_{1}\right|\left|z_{4}-z_{2}\right|^{p-1} .
\end{aligned}
$$

Therefore,

$$
\begin{aligned}
& \||u(t, \cdot+h)|^{p-1} u(t, \cdot+h)-|v(t, \cdot+h)|^{p-1} v(t, \cdot+h) \\
& \quad-|u(t)|^{p-1} u(t)+|v(t)|^{p-1} v(t) \|_{L^{r_{3}^{\prime}}\left(\mathbb{R}^{2}\right)} \\
& =\||u(t, \cdot+h)|^{p-1} u(t, \cdot+h)-|u(t)|^{p-1} u(t) \\
& \quad-|v(t, \cdot+h)|^{p-1} v(t, \cdot+h)+|v(t)|^{p-1} v(t) \|_{L^{\prime}{ }_{3}\left(\mathbb{R}^{2}\right)} \\
& \leq 4\|u(t)\|^{p-1}{ }^{\frac{2 r_{3}(p-1)}{r_{3}-2}}\left(\mathbb{R}^{2}\right) \\
& +\frac{2(p+2)}{p}\|v(t, \cdot+h)-v(t, \cdot+h)-u(t)+v(t)\|_{L^{2}\left(\mathbb{R}^{2}\right)}
\end{aligned}
$$

and this means

$$
\begin{aligned}
& \left\||u|^{p-1} u-|v|^{p-1} v\right\|_{L^{q_{3}^{\prime}}\left(0, T ; B_{r_{3}^{\prime}}^{s_{4}}\left(\mathbb{R}^{2}\right)\right)} \\
& \lesssim\|\| u\left\|_{L^{\frac{2 r_{3}(p-1)}{r_{3}-2}}\left(\mathbb{R}^{2}\right)}^{p-1}\right\| u-v\left\|_{H^{s_{4}}\left(\mathbb{R}^{2}\right)}+\right\| v\left\|_{H^{s_{4}}\left(\mathbb{R}^{2}\right)}\right\| u-v\left\|_{L^{\frac{2 r_{3}(p-1)}{r_{3}-2}}}^{p-1}\right\|_{\left.\mathbb{R}^{2}\right)} \|_{L^{q_{3}^{\prime}}(0, T)} \\
& \leq\|\| u\left\|_{L^{\infty}\left(\mathbb{R}^{2}\right)}^{p-1-\frac{r_{3}-2}{r_{3}}}\right\| u\left\|_{L^{2}\left(\mathbb{R}^{2}\right)}^{\frac{r_{3}-2}{r_{3}}}\right\| u-v\left\|_{H^{s_{4}\left(\mathbb{R}^{2}\right)}}\right\|_{L^{q_{3}^{\prime}(0, T)}}
\end{aligned}
$$

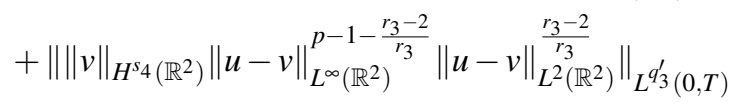

$$
\begin{aligned}
& \leq\|u\|_{L^{q_{3}^{\prime}\left(p-1-\frac{r_{3}-2}{r_{3}}\right)}\left(0, T ; L^{\infty}\left(\mathbb{R}^{2}\right)\right)}^{p-1-\frac{r_{3}-2}{r^{2}}}\|u\|_{L^{\infty}\left(0, T ; L^{2}\left(\mathbb{R}^{2}\right)\right)}^{\frac{r_{3}-2}{r_{3}}}\|u-v\|_{L^{\infty}\left(0, T ; H^{s_{4}}\left(\mathbb{R}^{2}\right)\right)}
\end{aligned}
$$

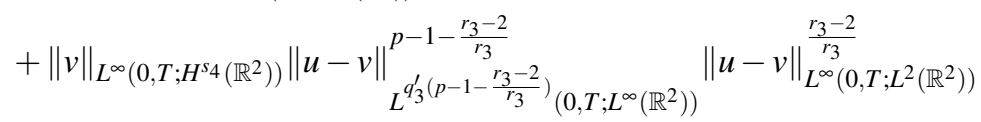

$$
\begin{aligned}
& \leq\|u\|_{L^{q_{1}\left(0, T ; L^{\infty}\left(\mathbb{R}^{2}\right)\right)}}^{p-1-\frac{r_{3}-2}{r_{3}}}\|u\|_{L^{\infty}\left(0, T ; L^{2}\left(\mathbb{R}^{2}\right)\right)}^{\frac{r_{3}-2}{r_{3}}}\|u-v\|_{L^{\infty}\left(0, T ; H^{s_{4}}\left(\mathbb{R}^{2}\right)\right)}
\end{aligned}
$$

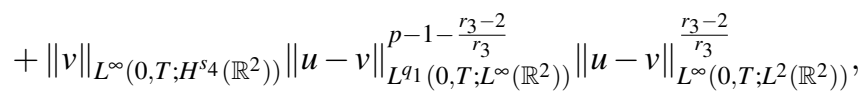

where $q_{1}, q_{3}>4>q_{3}^{\prime}>q_{3}^{\prime}\left(p-1-\frac{r_{3}-2}{r_{3}}\right)$. This and (22) imply that $u \rightarrow v$ in $L^{q_{1}}\left(0, T ; B_{r_{1}}^{s_{3}-\frac{3}{q_{1}}}\left(\mathbb{R}^{2}\right)\right)$ as $u_{0} \rightarrow v_{0}$ in $H^{s}\left(\mathbb{R}^{2}\right)$ because $u \rightarrow v$ in $\left(L^{\infty}\left(0, T ; L^{2}\left(\mathbb{R}^{2}\right)\right)\right)$ 
and $u, v$ are uniformly bounded in $\left(L^{\infty}\left(0, T ; H^{s}\left(\mathbb{R}^{2}\right)\right)\right)$ as $u_{0} \rightarrow v_{0}$ in $H^{s}\left(\mathbb{R}^{2}\right)$. Moreover,

$$
\begin{aligned}
& \|u-v\|_{L^{\infty}\left(0, T ; H^{s}\left(\mathbb{R}^{2}\right)\right)} \\
& \lesssim\left\|u_{0}-v_{0}\right\|_{H^{s}\left(\mathbb{R}^{2}\right)}+\left(\left\|u_{0}\right\|_{H^{s}\left(\mathbb{R}^{2}\right)}+\left\|v_{0}\right\|_{H^{s}\left(\mathbb{R}^{2}\right)}\right)\|u-v\|_{L^{p-1}\left(0, T ; L^{\infty}\left(\mathbb{R}^{2}\right)\right)}^{p-1} \\
& \left.\lesssim\left\|u_{0}-v_{0}\right\|_{H^{s}\left(\mathbb{R}^{2}\right)}+\left(\left\|u_{0}\right\|_{H^{s}\left(\mathbb{R}^{2}\right)}+\left\|v_{0}\right\|_{H^{s}\left(\mathbb{R}^{2}\right)}\right)\|u-v\|_{L^{q_{1}\left(0, T ; B_{r_{1}}\right.}}^{s_{3}-\frac{3}{q_{1}}}\left(\mathbb{R}^{2}\right)\right)
\end{aligned} .
$$

Therefore, the solution map is also continuously dependent in $L^{\infty}\left(0, T ; H^{s}\left(\mathbb{R}^{2}\right)\right)$.

\subsection{The case $n \geq 3$. Local $H^{1}$ existence result}

In the case where $n \geq 3$, the Strichartz estimate Lemma 1 doesn't seem sufficient to obtain a uniform control of solutions in the $H^{1}\left(\mathbb{R}^{3}\right)$ setting. So here, we consider radial data and use the following Strauss lemma.

Lemma 3 ([28, Theorems 1,2], [7, Proposition 1]). Let $n \geq 2$ and let $1 / 2<s<$ $n / 2$. Then for a radial function $f$

$$
\left\||\cdot|^{\frac{n}{2}-s} f\right\|_{L^{\infty}\left(\mathbb{R}^{n}\right)} \lesssim\|f\|_{\dot{H}_{\text {rad }}^{s}\left(\mathbb{R}^{n}\right)} .
$$

Since solutions are not uniformly controlled at the origin by the Strauss lemma above, we apply the following weighted Strichartz estimate:

Lemma 4 ([1, Propositions 2.2 and 2.3]). Let $n \in \mathbb{N}$. Let $\delta>0$ and $[x]_{\delta}=|x|^{1-\delta}+$ $|x|^{1+\delta}$. The for any $q_{1} \in[2, \infty]$ and $q_{2} \in(2, \infty]$,

$$
\begin{gathered}
\left\|[x]_{\delta}^{-1 / q_{1}} U(t) f\right\|_{L^{q_{1}\left(\mathbb{R} ; L^{2}\left(\mathbb{R}^{n}\right)\right)}} \lesssim\|f\|_{L^{2}\left(\mathbb{R}^{n}\right)}, \\
\left\|[x]_{\delta}^{-1 / q_{1}} \int_{0}^{t} U\left(t-t^{\prime}\right) F\left(t^{\prime}\right) d t^{\prime}\right\|_{L^{q_{1}\left(0, T ; L^{2}\left(\mathbb{R}^{n}\right)\right)}} \lesssim\left\|[x]_{\delta}^{1 / q_{2}} F\right\|_{L^{q_{2}^{\prime}\left(0, T ; L^{2}\left(\mathbb{R}^{n}\right)\right)}} .
\end{gathered}
$$

We can now prove Proposition 2.

Proof (Proof of Proposition 2). By using the uniform $H^{1}\left(\mathbb{R}^{n}\right)$ control obtained in (17), we reduce the proof to the local well-posedness in $H^{1}\left(\mathbb{R}^{n}\right)$. Let $\delta>0,1 / 2<$ $s<1$, and $2<q_{1}, q_{2}<\infty$ satisfy

$$
-(p-1)\left(\frac{n}{2}-s\right)+\frac{1-\delta}{q_{2}}=-\frac{1-\delta}{q_{1}} .
$$

We remark that there exist $\delta, q_{1}, q_{2}, s$ if $1<p<1+2 /(n-2)$ since,

$$
(p-1)\left(\frac{n}{2}-s\right)<1 \Longrightarrow p<1+\frac{2}{n-2 s}<1+\frac{2}{n-2} .
$$


We define the norm $Y^{1}(T)$ as

$$
\begin{aligned}
\|u\|_{Y^{1}(T)} & =\|u\|_{L^{\infty}\left(0, T ; H_{\mathrm{rad}}^{1}\left(\mathbb{R}^{n}\right)\right)} \\
& +\left\|[x]_{\delta}^{-1 / q_{1}} u\right\|_{L^{q_{1}\left(0, T ; L_{\mathrm{rad}}^{2}\left(\mathbb{R}^{n}\right)\right)}}+\left\|[x]_{\delta}^{-1 / q_{1}} \nabla u\right\|_{L^{q_{1}\left(0, T ; L_{\mathrm{rad}}^{2}\left(\mathbb{R}^{n}\right)\right)}} .
\end{aligned}
$$

Let $\psi \in \mathscr{S}\left(\mathbb{R}^{n} ;[0,1]\right)$ be radial and satisfy

$$
\psi(x)=\left\{\begin{array}{lll}
1 & \text { if } & |x| \leq 1 \\
0 & \text { if } & |x| \geq 2
\end{array}\right.
$$

Then by Lemmas 3 and 4 and (23),

$$
\begin{aligned}
& \|\Phi(u)\|_{Y^{1}(T)} \\
& \lesssim\left\|u_{0}\right\|_{H_{\mathrm{rad}}^{1}\left(\mathbb{R}^{n}\right)}+\left\|\int_{0}^{t} U\left(t-t^{\prime}\right)\left(\psi\left|u\left(t^{\prime}\right)\right|^{p-1} u\left(t^{\prime}\right)\right) d t^{\prime}\right\|_{Y^{1}(T)} \\
& +\left\|\int_{0}^{t} U\left(t-t^{\prime}\right)\left((1-\psi)\left|u\left(t^{\prime}\right)\right|^{p-1} u\left(t^{\prime}\right)\right) d t^{\prime}\right\|_{Y^{1}(T)} \\
& \lesssim\left\|u_{0}\right\|_{H_{\mathrm{rad}}^{1}\left(\mathbb{R}^{n}\right)} \\
& +\left.\left.\left\||x|^{-(p-1)\left(\frac{n}{2}-s\right)+\frac{1-\delta}{q_{2}}}\right\| x\right|^{\frac{n}{2}-s} u\right|^{p-1} u \|_{L^{q_{2}^{\prime}}\left(0, T ; L_{\mathrm{rad}}^{2}(|x| \leq 2)\right)} \\
& +\left.\left.\left\||x|^{-(p-1)\left(\frac{n}{2}-s\right)+\frac{1-\delta}{q_{2}}}\right\| x\right|^{\frac{n}{2}-s} u\right|^{p-1} \nabla u \|_{L^{\prime}{ }_{2}\left(0, T ; L_{\mathrm{rad}}^{2}(|x| \leq 2)\right)} \\
& +\left\||u|^{p-1} u\right\|_{L^{1}\left(0, T ; L_{\mathrm{rad}}^{2}(|x|>1)\right)}+\left\|\nabla\left(|u|^{p-1} u\right)\right\|_{L^{1}\left(0, T ; L_{\mathrm{rad}}^{2}(|x|>1)\right)} \\
& \lesssim\left\|u_{0}\right\|_{H_{\mathrm{rad}}^{1}\left(\mathbb{R}^{n}\right)}+T^{1-\frac{1}{q_{1}}-\frac{1}{q_{2}}}\|u\|_{Y^{1}(T)}^{p}
\end{aligned}
$$

and therefore for some $T$ and $R, \Phi$ is a map from $B_{Y^{1}(T)}(R)$ into itself. Moreover,

$$
\begin{aligned}
& \|\Phi(u)-\Phi(v)\|_{Y^{1}(T)} \\
& \lesssim\left\||x|^{-\frac{1-\delta}{q_{1}}}\left(\left.\left.|| x\right|^{\frac{n}{2}-s} u\right|^{p-1}-\left.\left.|| x\right|^{\frac{n}{2}-s} v\right|^{p-1}\right)(|\nabla u|+|u|)\right\|_{L^{q_{2}^{\prime}\left(0, T ; L_{\mathrm{rad}}^{2}(|x| \leq 2)\right)}} \\
& +\left.\left.|||x|^{-\frac{1-\delta}{q_{1}}}|| x\right|^{\frac{n}{2}-s} v\right|^{p-1}(|\nabla(u-v)|+|u-v|) \|_{L^{q_{2}^{\prime}\left(0, T ; L_{\mathrm{rad}}^{2}(|x| \leq 2)\right)}} \\
& +\left\|\left(\left.\left.|| x\right|^{\frac{n}{2}-s} u\right|^{p-1}-\left.\left.|| x\right|^{\frac{n}{2}-s} v\right|^{p-1}\right)|x|^{-\frac{1+\delta}{q_{1}}}(|\nabla u|+|u|)\right\|_{L^{1}\left(0, T ; L_{\text {rad }}^{2}(|x|>1)\right)} \\
& +\left.|||x|^{\frac{n}{2}-s} v\right|^{p-1}|x|^{-\frac{1+\delta}{q_{1}}}(|\nabla(u-v)|+|u-v|) \|_{L^{1}\left(0, T ; L_{\mathrm{rad}}^{2}(|x|>1)\right)} .
\end{aligned}
$$

Then for $p \geq 2, \Phi$ is a contraction map on $B_{Y^{1}(T)}(R)$. Similarly, for $1<p<2$, we define the auxiliary norm $Y^{0}(T)$ as

$$
\|u\|_{Y^{0}(T)}:=\|u\|_{L^{\infty}\left(0, T ; L_{\mathrm{rad}}^{2}\left(\mathbb{R}^{n}\right)\right)}+\left\|[x]_{\delta}^{-1 / q_{1}} u\right\|_{L^{q_{1}\left(0, T ; L_{\mathrm{rad}}^{2}\left(\mathbb{R}^{n}\right)\right)}} .
$$


Then for $1<p<2$,

$$
\begin{aligned}
& \|(\Phi(u)-\Phi(v))\|_{Y^{0}(T)} \\
& \lesssim \| \mid x]_{\delta}^{-1 / q_{1}}\left(\left.|| x\right|^{\frac{n}{2}-s} v|+||x|^{\frac{n}{2}-s} v \mid\right)^{p-1}|u-v| \|_{L^{q_{2}^{\prime}\left(0, T ; L_{\mathrm{rad}}^{2}(|x| \leq 2)\right)}} \\
& +\left\|\left(\left.|| x\right|^{\frac{n}{2}-s} v|+||x|^{\frac{n}{2}-s} v \mid\right)^{p-1}|u-v|\right\|_{L^{1}\left(0, T ; L_{\mathrm{rad}}^{2}(|x|>1)\right)} \\
& \lesssim T^{1-\frac{1}{q_{1}}-\frac{1}{q_{2}}}\left(\|u\|_{Y^{1}(T)}+\|v\|_{Y^{1}(T)}\right)^{p-1}\|u-v\|_{Y^{0}(T)}
\end{aligned}
$$

Therefore $\Phi$ is a contraction map on $Y^{0}(T)$ for some $T$ and $R$, which implies that (1) posses a unique solution in $Y^{1}(T)$. Moreover, by Lemma 3 and (25), with some $0<\theta<1$, for solutions $u$ and $v$ of (4) for initial data $u_{0}$ and $v_{0}$, respectively,

$$
\begin{aligned}
& \|u-v\|_{Y^{1}(T)} \\
& \lesssim\left\|u_{0}-v_{0}\right\|_{H_{\mathrm{rad}}^{1}\left(\mathbb{R}^{n}\right)}+T^{1-\frac{1}{q_{1}}-\frac{1}{q_{2}}}\left(\|u\|_{Y^{1}(T)}+\|v\|_{Y^{1}(T)}\right)^{p-1}\|u-v\|_{Y^{1}(T)} \\
& +T^{1-\frac{1}{q_{1}}-\frac{1}{q_{2}}}\left(\|u\|_{Y^{1}(T)}+\|v\|_{Y^{1}(T)}\right)\left\||x|^{\frac{n}{2}-s}(u-v)\right\|_{L^{\infty}\left(0, T ; L_{\text {rad }}^{\infty}\left(\mathbb{R}^{n}\right)\right)}^{p-1} \\
& \lesssim\left\|u_{0}-v_{0}\right\|_{H_{\mathrm{rad}}^{1}\left(\mathbb{R}^{n}\right)}+T^{1-\frac{1}{q_{1}}-\frac{1}{q_{2}}}\left(\|u\|_{Y^{1}(T)}+\|v\|_{Y^{1}(T)}\right)^{p-1}\|u-v\|_{Y^{1}(T)} \\
& +T\left(\|u\|_{Y^{1}(T)}+\|v\|_{Y^{1}(T)}\right)\|u-v\|_{Y^{1}(T)}^{p-1}
\end{aligned}
$$

and therefore $\|u-v\|_{Y^{1}(T)} \rightarrow 0$ as $\left\|u_{0}-v_{0}\right\|_{H_{\text {rad }}^{1}\left(\mathbb{R}^{n}\right)} \rightarrow 0$.

\subsection{Three dimensional case, small $H^{1}$ data solutions for $p=3$}

In the three dimensional scaling critical case, the weighted Strichartz estimate Lemma 4 doesn't seem sufficient to control solutions uniformly. So here, we transform (1) into the corresponding wave equation.

The Cauchy problem (1) with initial data $u(0)=u_{0}$ is rewritten as the following:

$$
\begin{aligned}
\square u & =i\left(-i \partial_{t}+D\right)|u|^{p-1} u \\
& =i \frac{p+1}{2}|u|^{p-1}\left(D u-i|u|^{p-1} u\right) \\
& -i \frac{p-1}{2}|u|^{p-3} u^{2} \overline{\left(D u-i|u|^{p-1} u\right)}+i D\left(|u|^{p-1} u\right) \\
& =i\left(D\left(|u|^{p-1} u\right)+\frac{p+1}{2}|u|^{p-1} D u-\frac{p-1}{2}|u|^{p-3} u^{2} D \bar{u}\right)+p|u|^{2 p-2} u \\
& =: F_{p}(u)
\end{aligned}
$$


Then the corresponding integral equation is the following:

$$
\begin{aligned}
u(t) & =\cos (t D) u_{0}+\frac{\sin (t D)}{D}\left(i D u_{0}+\left|u_{0}\right|^{p-1} u_{0}\right) \\
& +\int_{0}^{t} \frac{\sin \left(\left(t-t^{\prime}\right) D\right)}{D} F_{p}(u)\left(t^{\prime}\right) d t^{\prime} .
\end{aligned}
$$

For any radially symmetric function $f$, we define $\widetilde{f}$ as $\widetilde{f}(|x|)=f(x)$. Then for any radial data, (26) is rewritten as

$$
\widetilde{u}(t)=\partial_{t} J\left[u_{0}\right](t)+J\left[i D u_{0}+\left|u_{0}\right|^{p-1} u_{0}\right](t)+\int_{0}^{t} J\left[F_{p}(u)\left(t^{\prime}\right)\right]\left(t-t^{\prime}\right) d t^{\prime}
$$

where

$$
J[f](t, r)=\frac{1}{2 r} \int_{|r-t|}^{r+t} \lambda \widetilde{f}(\lambda) d \lambda .
$$

This transformation is justified as follows:

Lemma 5 ([12, Lemma 3.5]). Let $1<p \leq 3$ and $u_{0} \in H_{\mathrm{rad}}^{1}\left(\mathbb{R}^{3}\right)$ and $u \in C\left(0, T ; H_{\mathrm{rad}}^{1}\left(\mathbb{R}^{3}\right)\right)$ be the solution of (16). Then $u$ is also the solution of (27).

To obtain the uniform control, we use the estimates below regarding $J$. For any $f:[0, \infty) \rightarrow \mathbb{C}$, we define $A[f]: \mathbb{R} \rightarrow \mathbb{C}$ as $A[f](\lambda)=f(|\lambda|)$. See also [21].

Lemma $6([12$, Lemma 3.6]). Let $f:[0, \infty) \rightarrow \mathbb{C}$. Then

$$
\left\|\frac{1}{2 \cdot} \int_{|\cdot-t|}^{+t} f(\lambda) d \lambda\right\|_{L^{\infty}(0, \infty)} \leq M[A[f]](t),
$$

where $M$ is the Hardy-Littlewood-Maximal operator defined by

$$
M[h](x)=\sup _{r>0} \frac{1}{2 r} \int_{|x-y|<r}|h(y)| d y
$$

for $h: \mathbb{R} \rightarrow \mathbb{C}$.

Corollary 3 ([12, Corollary 3.7]). Let $f: \mathbb{R}^{3} \rightarrow \mathbb{C}$ be radial. Then

$$
\|J[f]\|_{L^{2}\left(0, T ; L^{\infty}\left(\mathbb{R}^{3}\right)\right)} \leq C\|f\|_{L_{\text {rad }}^{2}\left(\mathbb{R}^{3}\right)} .
$$

Corollary 4 ([12, Corollary 3.8]). Let $h:[0, \infty) \times \mathbb{R}^{3} \rightarrow \mathbb{C}$ be radial. Then

$$
\left\|\int_{0}^{t} J\left[h\left(t^{\prime}\right)\right]\left(t-t^{\prime}\right) d t^{\prime}\right\|_{L^{2}\left(0, T ; L^{\infty}(0, \infty)\right)} \leq C\|h\|_{L^{1}\left(0, T ; L_{\mathrm{rad}}^{2}\left(\mathbb{R}^{3}\right)\right)} .
$$

Corollary 5 (Hardy, [12, Corollary 3.9]). Let $f \in C^{1}([0, \infty)$; $\mathbb{C})$. Then

$$
\left\|\frac{d}{d t}\left(\frac{1}{2 r} \int_{|r-t|}^{r+t} \lambda f(\lambda) d \lambda\right)\right\|_{L^{2}\left(0, \infty ; L^{\infty}(0, \infty)\right)} \leq C\left\|r f^{\prime}\right\|_{L^{2}(0, \infty)} .
$$


Proof. Let $g$ be even extension of $f$.

$$
\begin{aligned}
\frac{d}{d t}\left(\frac{1}{2 r} \int_{|r-t|}^{r+t} \lambda f(\lambda) d \lambda\right) & =\frac{(r+t) f(r+t)-(t-r) f(|r-t|)}{2 r} \\
& =\frac{(r+t) f(r+t)-(t-r) g(t-r)}{2 r} \\
& =\frac{1}{2 r} \int_{-r}^{r}\left\{g(t+\tau)+(t+\tau) g^{\prime}(t+\tau)\right\} d \tau
\end{aligned}
$$

Then

$$
\left\|\frac{d}{d t}\left(\frac{1}{2 r} \int_{|r-t|}^{r+t} \lambda f(\lambda) d \lambda\right)\right\|_{L^{2}\left(0, \infty ; L^{\infty}(0, \infty)\right)} \leq\|M[g]\|_{L^{2}(\mathbb{R})}+\left\|M\left[\cdot g^{\prime}\right]\right\|_{L^{2}(\mathbb{R})} .
$$

Therefore, (28) and the following Hardy estimate([22, (0.2)]) imply Corollary 5 :

$$
\|g\|_{L^{2}(\mathbb{R})} \lesssim\left\|\cdot g^{\prime}\right\|_{L^{2}(\mathbb{R})} .
$$

We can now give the proof of Proposition 3.

Proof (Proof of Proposition 3). Let

$$
X_{\text {rad }}^{1}(0, T)=L^{\infty}\left(0, T ; H_{\text {rad }}^{1}\left(\mathbb{R}^{3}\right)\right) \cap L^{2}\left(0, T ; L_{\text {rad }}^{\infty}\left(\mathbb{R}^{3}\right)\right) .
$$

For $0<T<1$ and $p=3$, By Corollaries 3, 4, 5, and the Hölder and GagliardoNirenberg inequalities imply that, if initial data $u_{0}$ sufficiently small, then $\Phi$ maps $B_{X_{\mathrm{rad}}^{1}(0, T)}(R)$ into itself with some $T$ and $R$. Since

$$
\begin{aligned}
& \left|F_{3}(u)-F_{3}(v)\right| \\
& =\left.\left|i\left(D\left(|u|^{2} u\right)-2|u|^{2} D u-u^{2} D \bar{u}\right)+3\right| u\right|^{4} u \\
& -i\left(D\left(|v|^{2} v\right)-2|v|^{2} D v-v^{2} D \bar{v}\right)-3|v|^{4} v \mid \\
& \lesssim\left|D\left(|u|^{2} u-|v|^{2} v\right)\right|+|u|^{2}|D(u-v)| \\
& +\left(\left.|| u\right|^{2}-|v|^{2}|+| u^{2}-v^{2} \mid\right)|D v|+\left.|| u\right|^{4} u-|v|^{4} v \mid,
\end{aligned}
$$

we have

$$
\begin{aligned}
& \left\|F_{3}(u)-F_{3}(v)\right\|_{L^{1}\left(0, T ; L_{\mathrm{rad}}^{2}\left(\mathbb{R}^{3}\right)\right)} \\
& \lesssim\left(\|u\|_{X_{\mathrm{rad}}^{1}(0, T)}+\|v\|_{X_{\mathrm{rad}}^{1}(0, T)}\right)^{2}\|u-v\|_{X_{\mathrm{rad}}^{1}(0, T)} \\
& +\left(\|u\|_{X_{\mathrm{rad}}^{1}(0, T)}+\|v\|_{X_{\mathrm{rad}}^{1}(0, T)}\right)^{4}\|u-v\|_{X_{\mathrm{rad}}^{1}(0, T)} .
\end{aligned}
$$

This means $\Phi$ is a contraction map on $B_{X_{\text {rad }}^{1}(0, T)}(R)$ for sufficiently small $u_{0}$. 


\section{Blow-up for (1)}

At first, we recall the following ODE argument:

Lemma 7 ([11, Lemma 2.1]). Let $C_{1}, C_{2}>0$ and $q>1$. If $f \in C^{1}([0, T) ; \mathbb{R})$ satisfies $f(0)>0$ and

$$
f^{\prime}+C_{1} f=C_{2} f^{q} \quad \text { on }[0, T) \text { for some } T>0
$$

then

$$
f(t)=e^{-C_{1} t}\left(f(0)^{-(q-1)}+C_{1}^{-1} C_{2} e^{-C_{1}(q-1) t}-C_{1}^{-1} C_{2}\right)^{-\frac{1}{q-1}}
$$

Moreover, if $f(0)>C_{1}^{\frac{1}{q-1}} C_{2}^{-\frac{1}{q-1}}$, then $T<-\frac{1}{C_{1}(q-1)} \log \left(1-C_{1} C_{2}^{-1} f(0)^{-q+1}\right)$.

Next, we recall Calderón-Zygmund argument. We call $K$, a mesurable function on $\mathbb{R}^{n}$, Calderón-Zygmund (CZ) kernel if $K$ satisfies

$$
|K(x)| \leq|x|^{-n}, \quad|\nabla K(x)| \leq|x|^{-n+1}, \quad \int_{\varepsilon<|x|<R} K(x)=0, \quad 0<\forall \varepsilon<\forall R .
$$

Then CZ kernel is known to give a $L^{p}\left(\mathbb{R}^{n}\right)$ bounded operator as follows:

Lemma 8 ([3, Theorem 1]). Let $K$ be a CZ kernel. Then for $1<p<\infty$, there exists a positive constant $C$ such that

$$
\| \text { P.V. } \int_{\mathbb{R}^{n}} K(x-y) f(y) d y\left\|_{L^{p}\left(\mathbb{R}^{n}\right)} \leq C\right\| f \|_{L^{p}\left(\mathbb{R}^{n}\right)}
$$

for any $f \in L^{p}\left(\mathbb{R}^{n}\right)$.

Now we are in position to show Proposition 5.

Proof. Thanks to Lemma 7, it is enough to show

$$
\left\|\langle\cdot\rangle^{-q}\left[D,\langle\cdot\rangle^{q}\right]\right\|_{L^{2}\left(\mathbb{R}^{n}\right) \rightarrow L^{2}\left(\mathbb{R}^{n}\right)}<\infty .
$$

At first, We divide the operator into the following two pieces:

$$
\langle x\rangle^{-q}\left[(-\Delta)^{1 / 2},\langle x\rangle^{q}\right]=C T_{1}+C T_{2},
$$

where $\psi$ is a cut-off function defined by (24).

$$
\begin{aligned}
& T_{1}(f)(x)=\langle x\rangle^{-q} \int_{\mathbb{R}^{n}} \frac{(1-\psi(y))\left(\langle x\rangle^{q}-\langle x+y\rangle^{q}\right)}{|y|^{n+1}} f(x+y) d y, \\
& T_{2}(f)(x)=\langle x\rangle^{-q} \text { P.V. } \int_{\mathbb{R}^{n}} \frac{\psi(y)\left(\langle x\rangle^{q}-\langle x+y\rangle^{q}\right)}{|y|^{n+1}} f(x+y) d y .
\end{aligned}
$$

In order to estimate $T_{1}$ by dividing into two pieces: 


$$
T_{1}=T_{3}+T_{4},
$$

where

$$
\begin{aligned}
& T_{3}(f)(x)=\langle x\rangle^{-q} \int_{|x| \leq|y|} \frac{(1-\psi(y))\left(\langle x\rangle^{q}-\langle x+y\rangle^{q}\right)}{|y|^{n+1}} f(x+y) d y, \\
& T_{4}(f)(x)=\langle x\rangle^{-q} \int_{|x| \geq|y|} \frac{(1-\psi(y))\left(\langle x\rangle^{q}-\langle x+y\rangle^{q}\right)}{|y|^{n+1}} f(x+y) d y .
\end{aligned}
$$

By the Hölder and Young inequalities,

$$
\begin{aligned}
& \left\|T_{3}(f)\right\|_{L^{2}\left(\mathbb{R}^{n}\right)} \\
& \leq\left(1+2^{q}\right)\left\|\langle x\rangle^{-q} \int_{|x| \leq|y|} \frac{\langle y\rangle^{q}(1-\psi(y))}{|y|^{n+1}} f(x+y) d y\right\|_{L^{2}\left(\mathbb{R}^{n}\right)} \\
& \leq\left(1+2^{q}\right)\left\|\langle\cdot\rangle^{-q}\right\|_{L^{2}\left(\mathbb{R}^{n}\right)}\left\|\int_{\mathbb{R}^{n}} \frac{\langle y\rangle^{q}(1-\psi(y))}{|y|^{n+1}} f(x+y) d y\right\|_{L^{\infty}\left(\mathbb{R}^{n}\right)} \\
& \leq\left(1+2^{q}\right)\left\|\langle\cdot\rangle^{-q}\right\|_{L^{2}\left(\mathbb{R}^{n}\right)}\left\|\langle\cdot\rangle^{q}|\cdot|^{-n-1}(1-\psi)\right\|_{L^{2}\left(\mathbb{R}^{n}\right)}\|f\|_{L^{2}\left(\mathbb{R}^{n}\right)}
\end{aligned}
$$

Similarly by the Young inequality,

$$
\begin{aligned}
\left\|T_{4}(f)\right\|_{L^{2}\left(\mathbb{R}^{n}\right)} & \leq\left(1+2^{q}\right)\left\|\int_{\mathbb{R}^{n}} \frac{1-\psi(y)}{|y|^{n+1}}|f(x+y)| d y\right\|_{L^{2}\left(\mathbb{R}^{n}\right)} \\
& \leq\left(1+2^{q}\right)\left\||\cdot|^{-n-1}(1-\psi)\right\|_{L^{1}\left(\mathbb{R}^{n}\right)}\|f\|_{L^{2}\left(\mathbb{R}^{n}\right)} .
\end{aligned}
$$

Next, in order to estimate $T_{2}$, we recall that

$$
\begin{aligned}
\langle x+y\rangle^{q} & =\langle x\rangle^{q}+\frac{q}{2}\langle x\rangle^{q-2}\left(|x+y|^{2}-|x|^{2}\right)+R_{1}(x, y), \\
& =\langle x\rangle^{q}+q\langle x\rangle^{q-2} x \cdot y+R_{2}(x, y),
\end{aligned}
$$

where $R_{2}(x, y)=R_{1}(x, y)+q\langle x\rangle^{q-2}|y|^{2} / 2$ and

$$
R_{1}(x, y)=\frac{q(q-2)}{2^{2}} \int_{|x|^{2}}^{|x+y|^{2}}(1+\rho)^{q / 2-2}\left(|x+y|^{2}-\rho\right) d \rho .
$$

By combining (13) and (29), we have

$$
T_{2}=-q T_{5}-T_{6},
$$

where

$$
\begin{aligned}
& T_{5}(f)(x)=\frac{x}{\langle x\rangle^{2}} \cdot \mathrm{P} . \mathrm{V} \cdot \int_{\mathbb{R}^{n}} \frac{y \psi(y)}{|y|^{n+1}} f(x+y) d y, \\
& T_{6}(f)(x)=\frac{1}{\langle x\rangle^{q}} \text { P.V. } \int_{\mathbb{R}^{n}} \frac{R_{2}(x, y) \psi(y)}{|y|^{n+1}} f(x+y) d y .
\end{aligned}
$$


It is easy to see that $K(y)=y|y|^{-n-1} \psi(y)$ is a CZ kernel. Therefore

$$
\left\|T_{5}(f)\right\|_{L^{2}\left(\mathbb{R}^{n}\right)} \leq\left\|\mathrm{P} \cdot \mathrm{V} \cdot \int_{\mathbb{R}^{n}} \frac{y \psi(y)}{|y|^{n+1}} f(\cdot+y) d y\right\|_{L^{2}\left(\mathbb{R}^{n}\right)} \leq C\|f\|_{L^{2}\left(\mathbb{R}^{n}\right)} .
$$

Moreover, since

$$
\begin{aligned}
|y|^{-n-1}\left|R_{1}(x, y)\right| & \leq\left(\langle x\rangle^{q-2}+\langle x+y\rangle^{q-2}\right)\left(|x+y|^{2}-|x|^{2}\right)^{2}|y|^{-n-1} \\
& \leq\left(\langle x\rangle^{q-2}+\langle x+y\rangle^{q-2}\right)(|x+y|+|x|)^{2}|y|^{-n+1}
\end{aligned}
$$

by the Young inequality,

$$
\left\|T_{6}(f)\right\|_{L^{2}\left(\mathbb{R}^{n}\right)} \leq C\left\|\int_{\mathbb{R}^{n}} \frac{\psi(y)}{|y|^{n-1}} f(x+y) d y\right\|_{L^{2}\left(\mathbb{R}^{n}\right)} \leq\left\||\cdot|^{-n+1} \psi\right\|_{L^{1}\left(\mathbb{R}^{n}\right)}\|f\|_{L^{2}\left(\mathbb{R}^{n}\right)} .
$$

\section{A priori estimates}

This last section is devoted to the proofs of Propositions 7, 8, 9, 10, and 11. The proofs are essentially the same in [12], but we report them here for sake of completeness.

Proof (Proof of Proposition 7). The proposition follows from a standard argument, so we omit the proof.

Proof (Proof of Proposition 8). The proposition follows from a standard argument, so we omit the proof.

Proof (Proof of Proposition 9). Here we give a direct proof based on the integral equation by using the method in [27].

$$
\begin{aligned}
& \left\|u\left(t_{2}\right)\right\|_{\dot{H}^{s}\left(\mathbb{R}^{n}\right)}^{2} \\
& =\left\|u\left(t_{1}\right)\right\|_{\dot{H}^{s}\left(\mathbb{R}^{n}\right)}^{2}-2 \operatorname{Re} \int_{t_{1}}^{t_{2}}\left\langle D^{s}\left(|u(t)|^{p-1} u(t)\right), D^{s} u(t)\right\rangle_{L^{2}\left(\mathbb{R}^{n}\right)} d t \\
& \leq\left\|u\left(t_{1}\right)\right\|_{\dot{H}^{s}\left(\mathbb{R}^{n}\right)}^{2}+2 \int_{t_{1}}^{t_{2}}\left\|D^{s}\left(|u(t)|^{p-1} u(t)\right)\right\|_{L^{2}\left(\mathbb{R}^{n}\right)}\|u(t)\|_{\dot{H}^{s}\left(\mathbb{R}^{n}\right)} d t \\
& \leq\left\|u\left(t_{1}\right)\right\|_{\dot{H}^{s}\left(\mathbb{R}^{n}\right)}^{2}+C \int_{t_{1}}^{t_{2}}\|u(t)\|_{L^{\infty}\left(\mathbb{R}^{n}\right)}^{p-1}\|u(t)\|_{\dot{H}^{s}\left(\mathbb{R}^{n}\right)}^{2} d t,
\end{aligned}
$$

where we used the nonlinear estimate

$$
\left\||f|^{p-1} f\right\|_{\dot{H}^{s}\left(\mathbb{R}^{n}\right)} \lesssim\|f\|_{L^{\infty}\left(\mathbb{R}^{n}\right)}^{p-1}\|f\|_{\dot{H}^{s}\left(\mathbb{R}^{n}\right)}
$$

(see [13, Lemma 3.4]). 
Proof (Proof of Proposition 10). Since $|u|^{2} u \in C\left((0, T) ; H^{2}\left(\mathbb{R}^{n}\right)\right.$ ), the following calculation is justified by the Plancherel identity:

$$
\begin{aligned}
& \left\|u\left(t_{2}\right)\right\|_{\dot{H}^{2}\left(\mathbb{R}^{n}\right)}^{2} \\
& =\left\|u\left(t_{1}\right)\right\|_{\dot{H}^{2}\left(\mathbb{R}^{n}\right)}^{2}-2 \operatorname{Re} \int_{t_{1}}^{t_{2}}\left\langle\Delta|u(t)|^{2} u(t), \Delta u(t)\right\rangle_{L^{2}\left(\mathbb{R}^{n}\right)} d t \\
& =\left\|u\left(t_{1}\right)\right\|_{\dot{H}^{2}\left(\mathbb{R}^{n}\right)}^{2}-2 \operatorname{Re} \sum_{j, k=1}^{n} \int_{t_{1}}^{t_{2}}\left\langle|u(t)|^{2} \partial_{j} \partial_{k} u(t), \partial_{j} \partial_{k} u(t)\right\rangle_{L^{2}\left(\mathbb{R}^{n}\right)} d t \\
& -4 \operatorname{Re} \sum_{j, k=1}^{n} \int_{t_{1}}^{t_{2}}\left\langle\partial_{k} u(t) \partial_{j}|u(t)|^{2}, \partial_{j} \partial_{k} u(t)\right\rangle_{L^{2}\left(\mathbb{R}^{n}\right)} d t \\
& -2 \operatorname{Re} \sum_{j, k=1}^{n} \int_{t_{1}}^{t_{2}}\left\langle\partial_{j} \partial_{k}|u(t)|^{2}, \overline{u(t)} \partial_{j} \partial_{k} u(t)\right\rangle_{L^{2}\left(\mathbb{R}^{n}\right)} d t \\
& =\left\|u\left(t_{1}\right)\right\|_{\dot{H}^{2}\left(\mathbb{R}^{n}\right)}^{2}-2 \sum_{j, k=1}^{n} \int_{t_{1}}^{t_{2}}\left\|u(t) \partial_{j} \partial_{k} u(t)\right\|_{L^{2}\left(\mathbb{R}^{n}\right)}^{2} d t \\
& +2 \sum_{j, k=1}^{n} \int_{t_{1}}^{t_{2}}\left\langle\partial_{j}^{2}|u(t)|^{2},\left|\partial_{k} u(t)\right|^{2}\right\rangle_{L^{2}\left(\mathbb{R}^{n}\right)} d t \\
& -\sum_{j, k=1}^{n} \int_{t_{1}}^{t_{2}}\left\langle\partial_{j} \partial_{k}|u(t)|^{2}, \partial_{j} \partial_{k}|u(t)|^{2}-2 \operatorname{Re}\left(\overline{\partial_{j} u(t)} \partial_{k} u(t)\right)\right\rangle_{L^{2}\left(\mathbb{R}^{n}\right)} d t .
\end{aligned}
$$

By the Hölder,Young, and Sobolev inequalities,

$$
\begin{aligned}
& \left\|u\left(t_{2}\right)\right\|_{\dot{H}^{2}\left(\mathbb{R}^{n}\right)}^{2} \\
& \leq\left\|u\left(t_{1}\right)\right\|_{\dot{H}^{2}\left(\mathbb{R}^{n}\right)}^{2}-2 \sum_{j, k=1}^{n} \int_{t_{1}}^{t_{2}}\left\|u(t) \partial_{j} \partial_{k} u(t)\right\|_{L^{2}\left(\mathbb{R}^{n}\right)}^{2} d t \\
& +2 n^{2} \sum_{k=1}^{n} \int_{t_{1}}^{t_{2}}\left\|\partial_{k} u(t)\right\|_{L^{4}\left(\mathbb{R}^{n}\right)}^{4} d t+2 \sum_{j, k=1}^{n} \int_{t_{1}}^{t_{2}}\left\|\partial_{j} u(t)\right\|_{L^{4}\left(\mathbb{R}^{n}\right)}^{2}\left\|\partial_{k} u(t)\right\|_{L^{4}\left(\mathbb{R}^{n}\right)}^{2} d t \\
& \leq\left\|u\left(t_{1}\right)\right\|_{\dot{H}^{2}\left(\mathbb{R}^{n}\right)}^{2}-2 \sum_{j, k=1}^{n} \int_{t_{1}}^{t_{2}}\left\|u(t) \partial_{j} \partial_{k} u(t)\right\|_{L^{2}\left(\mathbb{R}^{n}\right)}^{2} d t \\
& +2 n^{2}(n+1) \int_{t_{1}}^{t_{2}}\|u(t)\|_{\dot{H}^{1}\left(\mathbb{R}^{n}\right)}^{4-n}\|u(t)\|_{\dot{H}^{2}\left(\mathbb{R}^{n}\right)}^{n} d t .
\end{aligned}
$$

We can now conclude the paper by showing Proposition 11.

Proof (Proof of Proposition 11). When $s=1$ and when $s=2$ and $p=3$, a priori estimates shows the global well-posedness by the blow-up alternative argument. Here we consider the case where $p=3$ and $1<s<2$. Let $[a]$ be the floor function 
of $a$. Let $T_{1}=\min \left\{1, T_{0}\right\}$. By using the $H^{1}$ a priori estimate, for any $t>0$,

$$
\begin{aligned}
\|u\|_{L^{4}\left(0, t ; L^{\infty}\left(\mathbb{R}^{2}\right)\right)} & \leq \sum_{k=0}^{\left[t / T_{1}\right]+1}\|u\|_{L^{4}\left(k T_{1},(k+1) T_{1} ; L^{\infty}\left(\mathbb{R}^{2}\right)\right)} \\
& \leq \sum_{k=0}^{\left[t / T_{1}\right]+1}\|u\|_{X^{1}\left(k T_{1},(k+1) T_{1}\right)} \\
& \leq 2 T_{1}^{-1}(1+t)\left\|u_{0}\right\|_{H^{1}\left(\mathbb{R}^{2}\right)}
\end{aligned}
$$

Then by using Proposition 10,

$$
\begin{aligned}
\|u(t)\|_{\dot{H}^{s}\left(\mathbb{R}^{2}\right)}^{2} & \lesssim\left\|u_{0}\right\|_{H^{s}\left(\mathbb{R}^{2}\right)}^{2}+\int_{0}^{t}\left\|u\left(t^{\prime}\right)\right\|_{L^{\infty}\left(\mathbb{R}^{n}\right)}^{2}\left\|u\left(t^{\prime}\right)\right\|_{\dot{H}^{s}\left(\mathbb{R}^{2}\right)}^{2} d t \\
& \lesssim\left\|u_{0}\right\|_{H^{s}\left(\mathbb{R}^{2}\right)}^{2}+\left\|u\left(t^{\prime}\right)\right\|_{L^{4}\left(0, t ; L^{\infty}\left(\mathbb{R}^{2}\right)\right)}^{2}\|u\|_{L^{4}\left(0, t ; \dot{H}^{s}\left(\mathbb{R}^{2}\right)\right)}^{2} \\
& \lesssim\left\|u_{0}\right\|_{H^{s}\left(\mathbb{R}^{2}\right)}^{2}+\left\|u_{0}\right\|_{H^{1}\left(\mathbb{R}^{2}\right)}^{2}(1+t)^{2}\|u\|_{L^{4}\left(0, t ; \dot{H}^{s}\left(\mathbb{R}^{2}\right)\right)}^{2}
\end{aligned}
$$

This shows

$$
\|u(t)\|_{\dot{H}^{s}\left(\mathbb{R}^{2}\right)}^{4} \lesssim\left\|u_{0}\right\|_{H^{s}\left(\mathbb{R}^{2}\right)}^{4}+\left\|u_{0}\right\|_{H^{1}\left(\mathbb{R}^{2}\right)}^{4}(1+t)^{4}\|u\|_{L^{4}\left(0, t ; \dot{H}^{s}\left(\mathbb{R}^{2}\right)\right)}^{4} .
$$

Therefore Gronwall inequality imply the global well-posedness in $H^{s}\left(\mathbb{R}^{2}\right)$.

Acknowledgements V. Georgiev was supported in part by INDAM, GNAMPA - Gruppo Nazionale per l'Analisi Matematica, la Probabilità e le loro Applicazioni, by Institute of Mathematics and Informatics, Bulgarian Academy of Sciences and Top Global University Project, Waseda University and the Project PRA 2018 - 49 of University of Pisa. The authors are grateful to the referees for their helpful comments.

\section{References}

1. Bellazzini, J., Georgiev, V., Visciglia, N.: Long time dynamics for semirelativistic NLS and half wave in arbitrary dimension. Math. Annalen. 371, no. 1-2, 707-740 (2018)

2. Borgna, J. P., Rial, D. F.: Existence of ground states for a one-dimensional relativistic Schödinger equation. J. Math. Phys. 53, 062301 (2012)

3. Calderón, A. P., Zygmund, A.: On the existence of certain singular integrals. Acta Math. 88, 85-139 (1952)

4. Cazenave, T.: Semilinear Schrödinger equations. Courant Lecture Notes in Mathematics. 10. American Mathematical Society. New York University. Courant Institute of Mathematical Sciences, New York; American Mathematical Society. Providence. RI. (2003)

5. Cazenave, T., Weissler, F.B.: Some remarks on the nonlinear Schrödinger equation in the critical case Nonlinear semigroups, partial differential equations and attractors (Washington, DC, 1987). Lecture Notes in Math. 1394, 18-29, Springer, Berlin (1989)

6. Cazenave, T., Weissler, F.B.: The Cauchy problem for the critical nonlinear Schrödinger equation in $H^{s}$. Nonlinear Analysis. Theory, Methods \& Applications. An International Multidisciplinary Journal. Series A: Theory and Methods. 14, 807-836 (1990) 
7. Cho, Y., Ozawa, T.: Sobolev inequalities with symmetry. Commun. Contemp. Math. 11, 355365 (2009)

8. Di Nezza, E., Palatucci, G., Valdinoci, E.: Hitchhikers guide to the fractional Sobolev spaces. Bull. Sci. Math.. 136, 521-573 (2012)

9. Forcella, L., Fujiwara, K., Georgiev, V., Ozawa, T.: Local well-posedness and blow-up for the half Ginzburg-Landau-Kuramoto equation with rough coefficients and potential. arXiv:1804.02524 (2018)

10. Fujiwara, K.: Remark on local solvability of the Cauchy problem for semirelativistic equations. J. Math. Anal. Appl. 432, 744-748 (2015)

11. Fujiwara, K., Georgiev, V., Ozawa, T.: Blow-up for self-interacting fractional GinzburgLandau equation. Dyn. Partial Differ. Equ. 15, 175-182 (2018)

12. Fujiwara, K., Georgiev,V., Ozawa, T.: On global well-posedness for nonlinear semirelativistic equations in some scaling subcritical and critical cases. arXiv:1611.09674 (2016)

13. Ginibre, J., Ozawa, T., Velo, G.: On the existence of the wave operators for a class of nonlinear Schrödinger equations. Ann. Inst. H. Poincaré Phys. Théor., 60, 211-239 (1994)

14. Ginibre, J., Velo, G.: Generalized Strichartz inequalities for the wave equation. J. Funct. Anal. 133, 50-68 (1995)

15. Grafakos, L.,Oh, S.: The Kato-Ponce Inequality, Comm. Partial Differential Equations 39, 1128-1157 (2014)

16. Ikeda, M., Inui, T.: Some non-existence results for the semilinear Schrödinger equation without gauge invariance. J. Math. Anal. Appl. 425, 758-773 (2015)

17. Ikeda, M., Wakasugi, Y.: Small-data blow-up of $L^{2}$-solution for the nonlinear Schrödinger equation without gauge invariance. Differential Integral Equations 26, 11-12 (2013)

18. Inui, T.: Some nonexistence results for a semirelativistic Schrödinger equation with nongauge power type nonlinearity. Proc. Amer. Math. Soc. 144, 2901-2909 (2016)

19. Kato T.,Ponce, G. Commutator estimates and the Euler and Navier-Stokes equations. Comm. Pure Appl. Math. 41, 891-907 (1988)

20. Kenig, C., Ponce, G., Vega, L.: The Cauchy problem for the Korteweg-de Vries equation in Sobolev spaces of negative indices, Duke Math. J. 71, 1-21 (1993)

21. Klainerman, S., Machedon, M.: Space-time estimates for null forms and the local existence theorem. Comm. Pure Appl. Math. 46, 1221-1268 (1993)

22. Kufner, A., Opic, B.: Hardy-type Inequalities. Pitman research notes in mathematics series. Longman Scientific \& Technical. (1990)

23. Laskin N.: Fractional quantum mechanics and Lévy path integrals. Physics Letters A. 268, 298-305 (2000)

24. Lenzmann, E., Schikorra, A.: Sharp commutator estimates via harmonic extensions. arXiv: 1609.08547

25. Li, D.: On Kato-Ponce and fractional Leibniz. Rev. Mat. Iberoamericana. to appear. arXiv:1609.01780v2

26. Nakamura, M., Ozawa, T.: The Cauchy problem for nonlinear Klein-Gordon equations in the Sobolev spaces. Publ. Res. Inst. Math. Sci. 37, 255-293 (2001)

27. Ozawa, T.: Remarks on proofs of conservation laws for nonlinear Schrödinger equations. Calc. Var. Partial Differential Equations. 25, 403-408 (2006)

28. Sickel, W., Skrzypczak, L.: Radial subspaces of Besov and Lizorkin-Triebel classes: extended Strauss lemma and compactness of embeddings. J. Fourier Anal. Appl. 6, 639-662 (2000) 